\title{
Induction of apoptosis in nasal polyp-derived fibroblasts by bleomycin A5 in vitro
}

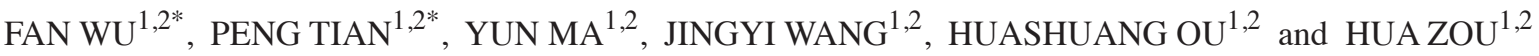 \\ ${ }^{1}$ Department of Otorhinolaryngology-Head and Neck Surgery; ${ }^{2}$ Guangdong Provincial Key Laboratory of \\ Malignant Tumor Epigenetics and Gene Regulation, Sun Yat-sen Memorial Hospital, \\ Sun Yat-sen University, Guangzhou, Guangdong 510000, P.R. China
}

Received October 8, 2017; Accepted January 17, 2018

DOI: $10.3892 / \mathrm{mmr} .2018 .8540$

\begin{abstract}
The present study aimed to evaluate the pro-apoptotic effects of bleomycin A5 on nasal polyp-derived fibroblasts (NPDFs) and the underlying molecular mechanisms. Nasal polyp tissue was acquired from 10 patients during surgery and NPDFs were isolated from surgical tissues. Fibroblasts were identified using immunohistochemistry. Bleomycin A5 was used to treat NPDFs along a concentration gradient. Cell viability was evaluated using a Cell Counting Kit-8 assay. A flow cytometric Annexin V-fluorescein isothiocyanate/propidium iodide assay was used to determine the percentage of apoptotic NPDFs. The mRNA expression levels of apoptotic genes were determined by reverse transcription-quantitative polymerase chain reaction and levels of proteins associated with apoptosis were determined by western blotting. The results indicated that bleomycin A5 was able to induce apoptosis in NPDFs in a dose-dependent manner. NPDFs treated with bleomycin A5 were identified to contain significantly high amounts of the active forms of caspase- 3 and showed considerable cleavage of poly(ADP-ribose) polymerase. The mRNA and protein expression levels of the pro-apoptotic molecule Bcl-2-associated X protein were significantly higher in treated NPDFs than in untreated NPDFs. In contrast, the mRNA and protein expression of the anti-apoptotic molecule B-cell lymphoma 2 (Bcl-2) was significantly lower in treated NPDFs. These results indicated that bleomycin A5 could induce apoptosis in primary NPDFs through activation of the Bcl-2 family and caspase cascades in a time-, and concentration-dependent manner.
\end{abstract}

Correspondence to: Professor Hua Zou, Department of Otorhinolaryngology-Head and Neck Surgery, Sun Yat-sen Memorial Hospital, Sun Yat-sen University, 107 Yan Jiang Xi Road, Guangzhou, Guangdong 510000, P.R. China

E-mail: zouhua28@163.com

*Contributed equally

Key words: nasal polyps, fibroblasts, bleomycin A5, apoptosis

\section{Introduction}

Nasal polyps are a heterogeneous disease characterized by predominant infiltration of inflammatory cells, structural fibrosis, edematous stromal tissue, and thickening of the basement membrane (1). The prevalence is about $4 \%$ (2). Much of the nasal polyp stroma is dense with fibroblasts, which produce many kinds of cytokines for polymorphonuclear leukocytes. The fibroblasts in nasal polyp tissue can be transformed into myofibroblasts, which are the main source of extracellular matrix in nasal polyps $(3,4)$. This suggests the importance of controlling fibroblast functions, such as proliferation, collagen, and cytokine production, in the management and treatment of nasal polyps $(5,6)$.

Bleomycin A5 is a cytotoxic antibiotic derived from Streptomyces verticellus. It has antineoplastic, antibacterial, and antiviral properties. Intralesional bleomycin A5 has recently come to be used in the treatment of keloid and hypertrophic scars $(7,8)$. Several studies have besen performed to explain the exact mechanism by which bleomycin A5 resolves keloids and hypertrophic scars. Some researchers have shown that cultured human dermal fibroblasts treated with bleomycin A5 can diminish collagen synthesis (9). Similarly, administration of bleomycin A5 to cultured fibroblasts causes a reduction in lysyl oxidase levels (10). It has also been reported that bleomycin A5 induces apoptosis in epithelial cells and fibroblasts in the lungs through production of reactive oxygen species (ROS), and activation of the Fas-FasL associated caspase cascade-mediated pathway $(11,12)$.

Recently, intralesional bleomycin A5 injections have been used to treat nasal polyps in China. It has also been reported that bleomycin A5 induces apoptosis among eosinophils in nasal polyp tissue (13). Based on previous studies, it is here hypothesized that bleomycin A5 may also have a pro-apoptotic effect on NPDFs. For this reason, this study was designed to ascertain whether the apoptotic signaling pathway of bleomycin A5 becomes activated in NPDFs.

\section{Materials and methods}

Isolation, culture, and identification of fibroblasts. Here, 10 patients ( 6 females and 4 males, $36.3 \pm 4.1$ years) who were non-smokers and had either not been treated with 
glucocorticoids (systemic or topical), antihistamines, non-steroidal anti-inflammatory drugs, or macrolide antibiotics for at least 1 month or who had ceased using the drug at least 1 month due to lack of alleviation or even aggravation of symptoms, were recruited from the Department of Otorhinolaryngology at Sun Yat-sen Memorial Hospital. All participants provided written informed consent in advance, and nasal polyp tissues were obtained during surgery. The study was approved by the Ethics Committee of Sun Yat-sen Memorial Hospital.

NPDFs were isolated from nasal polyp tissue from patients who had chronic rhinosinusitis with nasal polyps using a previously described method (14). Briefly, nasal polyp tissue was cut into pieces, re-suspended in DMEM/F12 containing $1 \mathrm{mg} / \mathrm{ml}$ collagenase $\mathrm{I}$ in a centrifuge tube, and then placed in a $5 \% \mathrm{CO}_{2}$ humidified atmosphere at $37^{\circ} \mathrm{C}$. After $12 \mathrm{~h}$, the tissues were re-suspended in DMEM/F12 with $10 \% \mathrm{FBS}$ and cultured in a $5 \% \mathrm{CO}_{2}$-humidified atmosphere at $37^{\circ} \mathrm{C}$, and the medium was changed every $48 \mathrm{~h}$. Trypsin enzymatic digestion and differential attachment were used to isolate fibroblast cells .

The isolated fibroblasts were identified immunocytochemistry using vimentin as a positive marker and CK (pan) as a negative marker. After 3 rounds of washing with PBS, the cells were incubated for $30 \mathrm{~min}$ with $5 \%$ BSA to block non-specific sites. The coverslips were then incubated with primary antibody (vimentin or $\mathrm{CK}$ ) (1:200 dilution) in $1 \%$ BSA in a humidified chamber overnight at $4^{\circ} \mathrm{C}$. Then the coverslips were incubated with HRP conjugated anti-rabbit secondary antibody for $30 \mathrm{~min}$, and washed 3 times for 5 min each with PBS. DAB substrate was used to detect HRP activity. Another group of NPDFs were grown in 6-well plates using DMEM/F12 containing 10\% FBS with or without 100 , 200 , or $400 \mu \mathrm{M}$ bleomycin $\mathrm{A} 5$ for $48 \mathrm{~h}$ in a $5 \% \mathrm{CO}_{2}$ humidified atmosphere at $37^{\circ} \mathrm{C}$ to assess the influence of bleomycin A5 on NPDFs.

CCK- 8 array of NPDFs. Cultured NPDFs $\left(1 \times 10^{4}\right)$ were seeded in each well of a 96-well plate in triplicate. After attachment, the cells were treated with bleomycin A5 at various concentrations (from 0 to $400 \mu \mathrm{M}$ at $50 \mu \mathrm{M}$ intervals) for 24,48 , or $72 \mathrm{~h}$. Then cells were incubated for $3 \mathrm{~h}$ with CCK-8 reagent (100 $\mu \mathrm{l} / \mathrm{ml}$ medium) (Dojindo Molecular Technologies, Inc., Kumamoto, Japan). Absorbance was determined at $490 \mathrm{~nm}$ using a microplate reader (Thermo Fisher Scientific Inc., Cramlington, UK).

Flow cytometric Annexin V-fluorescein isothiocyanate (FITC)/propidium iodide assay of fibroblast cells. An Annexin V/PI Apoptosis kit (Thermo Fisher Scientific Inc.) was used to assess the rate of apoptosis of fibroblast cells after bleomycin A5 treatment in accordance with the manufacturer's instructions. Briefly, after incubation in 6-well plates with DMEM/F12 with $10 \%$ FBS medium in the presence of various concentrations of $\operatorname{BLE}(0,100$, 200 or $400 \mu \mathrm{M}$ ) for $48 \mathrm{~h}$, the cultured fibroblast cells were gently suspended in binding buffer and incubated for $15 \mathrm{~min}$ at room temperature in the dark with $5 \mu \mathrm{l}$ Annexin V-FITC and $10 \mu \mathrm{l} \mathrm{PI}$. The Annexin V-FITC- and PI-labelled cells were analyzed using a flow cytometer (BD Biosciences, Burlington, MA, USA). Using flow cytometry, dot plots of
PI on the $y$-axis, against Annexin V-FITC on the $\mathrm{x}$-axis were used to distinguish viable cells, which are negative for PI and Annexin V-FITC, cells in the early stages of apoptosis (Annexin V-positive/PI-negative), and cells in late apoptosis or full necrosis (Annexin V-FITC-positive/PI-positive staining).

Reverse transcription-quantitative polymerase chain reaction. After bleomycin $\mathrm{A} 5$ treatment at various concentrations (including 0,100, 200, and $400 \mu \mathrm{M}$ ), fibroblasts were homogenized in $1 \mathrm{ml}$ TRIzol reagent (Invitrogen, Carlsbad, CA, USA). Total RNA was obtained, and $2 \mu \mathrm{g}$ RNA/cell sample was reverse transcribed into complementary DNA (cDNA) using the reverse transcription system (Toyobo Co., Ltd., Osaka, Japan). Quantitative polymerase chain reaction was performed using Roche Light cycler 96 system (Roche Diagnostics, Basel, Switzerland) with a $10 \mu$ l volume mixture containing $2 \mu \mathrm{l}$ cDNA, $0.2 \mu \mathrm{l}$ of each primer, and $5 \mu \mathrm{l}$ SYBR-Green (Toyobo Co., Ltd.). The relative mRNA expression levels of apoptosis-related genes were compared using the $2^{-\Delta \Delta \mathrm{Cq}}$ method. The sequences of the primers used for PCR were ascertained from the primer bank and are listed as follows: GADPH forward, 5'-CAGTGCCAGCCTCTGCTC AT-3' and reverse, 5'-ATACTCAGCACCAGCACAT-3'; Bcl-2 forward, 5'-CTGGGATGCCTTTGTGGAAC-3' and reverse, 5'-GGCAGGCATGTTGACTTCAC-3'; Bax forward, 5'-CCA AGAAGCTGAGCGAGTGT-3' and reverse, 5'-CAGCCCATG ATGGTTCTGAT-3'; caspase-3 forward, 5'-ATGCAGCAA ACCTCAGGGAA-3' and reverse, 5'-GTCGGCCTCCACTGG TATTT-3'.

Western blot analysis. NPDFs treated with bleomycin A5 were homogenized in RIPA buffer (Sigma-Aldrich;. Merck KGaA, Darmstadt, Germany) on ice for $30 \mathrm{~min}$. Using a BCA protein assay kit (Beyotime Institute of Biotechnology, Beijing, China), equal amounts of proteins were separated using $10 \%$ sodium dodecyl sulfate-polyacrymide (SDS) gel electrophoresis and transferred onto polyvinylidene fluoride membranes (Milipore, Billerica, MA, USA). They were then blocked using 5\% non-fat milk in TBS-0.1\% Tween-20 for $1 \mathrm{~h}$ at room temperature. The membranes were then incubated overnight at $4^{\circ} \mathrm{C}$ with primary antibodies for caspase-3 (1:500 dilution), cleaved caspase-3 (1:1,000 dilution), poly(ADP-ribose) polymerase (PARP) (1:1,000 dilution), active PARP (1:1,000 dilution), $\operatorname{Bax}$ (1:2,000 dilution), Bcl-2 (1:1,000 dilution), or GADPH (1:10,000 dilution) (Santa Cruz Biotechnology, Inc., Santa Cruz, CA, USA). After washing in TBST, the membranes were incubated with horseradish-peroxidase-conjugated anti-rabbit secondary antibodies for $1 \mathrm{~h}$ at room temperature and then visualized using an enhanced chemiluminescence kit (Millipore, Billerica, MA, USA). Images of the bands were captured using a Bio-Rad Gel Doc XR documentation system (Bio-Rad Laboratories, Inc., Hercules, CA, USA). The levels of relative protein expression were determined by densitometry and standardized to the GAPDH levels using ImageJ software (National Institutes of Health, Bethesda, MD, USA).

Bax gene silencing by siRNA. Mitochondria-mediated caspase-dependent pro-apoptotic protein Bax was inhibited using small interfering RNA (siRNA). Cells $\left(2.5 \times 10^{5} /\right.$ well) were plated in 6-well plates and transfected with $20 \mathrm{nM}$ 

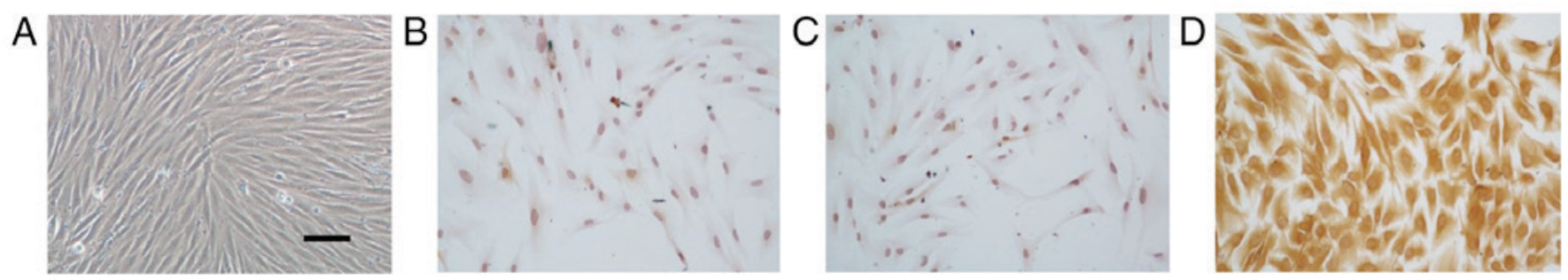

Figure 1. (A) NPDFs morphology observed by optical microscope. (B-D) Immunohistochemical staining to identify NPDFs using PBS as control group (B) CK (pan) as a negative marker (C) and vimentin as a positive marker (D). Scale bars, $50 \mu \mathrm{m}$. Original magnification, x400. NPDFs, nasal polyp-derived fibroblasts.

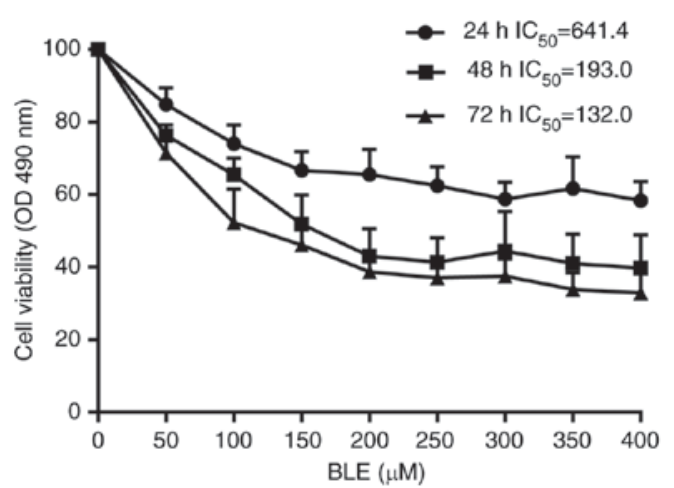

Figure 2. NPDFs were exposed to various concentrations of bleomycin A5 for 24,48 , and $72 \mathrm{~h}$ with 3 wells/group. Cell viability curves were plotted as viable cell percentage with \pm SD based on the CCK -8 assay. NPDFs, nasal polyp-derived fibroblasts.

Bax-targeting siRNA or control siRNA (Santa Cruz Biotechnology, Inc.) using Lipofectamine 3000 kit (Life Technologies, Japan) according to the manufacturer's instructions and cultured for $72 \mathrm{~h}$ at $37^{\circ} \mathrm{C}$ in a $5 \% \mathrm{CO}_{2}$ incubator. The cells were then analyzed for Bax protein levels by western blot analysis using an anti-Bax antibody. The membranes were re-probed with an anti-GAPDH antibody to verify equal loading. Then, transfected NPDFs were treated with $200 \mu \mathrm{M}$ BLE for $48 \mathrm{~h}$ and analyzed for apoptosis by Annexin V/PI staining in a flow cytometer. Annexin $\mathrm{V}^{+}$cells were considered to be apoptotic cells.

Statistical analysis. All experiments were performed 3 times to provide sufficient data. The differences between the experimental conditions and the controls were analyzed through one-way analysis of variance and Tukey's test (GraphPad Prism, version 5; GraphPad Software, San Diego, CA, USA). Statistical significance was determined at the $95 \%$ confidence level. P-values $<0.05$ were considered to indicate a statistically significant difference.

\section{Results}

Identification of NPDFs. To identify NPDFs, we first isolated fibroblast cells from nasal polyp tissue observe the cell morphologies. As showed in Fig. 1A, NPDFs were spindle and nest-like distributed. Immunocytochemistry staining of primary NPDFs indicated that the isolated and cultured cells were vimentin-positive and CK (pan)-negative, which was consistent with the characteristics of fibroblasts (Fig. 1B-D).
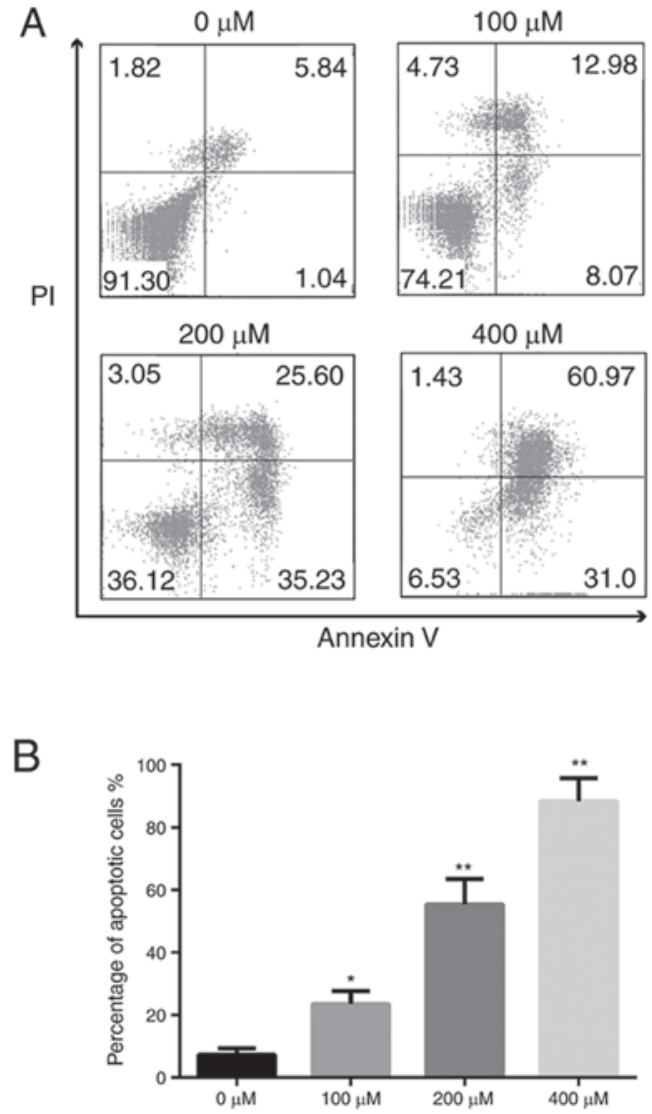

Figure 3. (A) Dot plots of Annexin-V/PI flow cytometric analysis. (B) Bar graphs of relative number of apoptotic cells. The results are here expressed as the mean \pm standard deviation $(\mathrm{n}=3)$. ${ }^{*} \mathrm{P}<0.05$ and ${ }^{* *} \mathrm{P}<0.001$, relative to the control group.

Bleomycin A5 induces NPDF apoptosis in a concentrationand time-dependent manner. Next, we examined the effect of bleomycin A5 on NPDF viability. Results showed that groups treated with higher concentrations of bleomycin A5 had lower cell viability. This phenomenon was especially visible at doses ranging from 0 to $200 \mu \mathrm{M}$. As the duration of bleomycin A5 exposure increased, cell viability also decreased in all treated groups. In the $48 \mathrm{~h}$ group, the $\mathrm{IC}_{50}=193.0$ (95\% confidence intervals, 146.3 to 254.5 ) (Fig. 2). These results indicated that the effect of bleomycin A5 on NPDFs was concentration- and time-dependent.

Flow cytometry indicated that the ratio of apoptotic cells was $7.25 \pm 2.09 \%$ in the $0 \mu \mathrm{M}$ group, $23.50 \pm 4.09 \%$ in $100 \mu \mathrm{M}$ group, $55.27 \pm 8.17 \%$ in the $200 \mu \mathrm{M}$ group, and $88.32 \pm 7.39 \%$ in $400 \mu \mathrm{M}$ group (Fig. 3). NPDFs exposed to bleomycin A5 


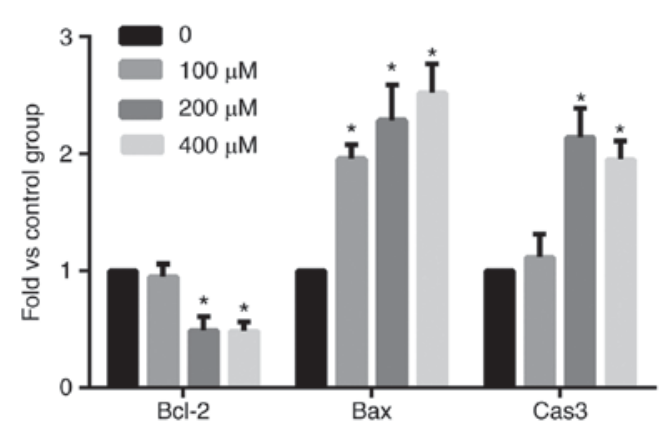

Figure 4. mRNA levels of genes involved in apoptosis of NPDFs induced by different doses of bleomycin A5 for $48 \mathrm{~h}$ using RT-qPCR. " $\mathrm{P}<0.05$ relative to the control group $(\mathrm{n}=3)$. NPDFs, nasal polyp-derived fibroblasts.

A

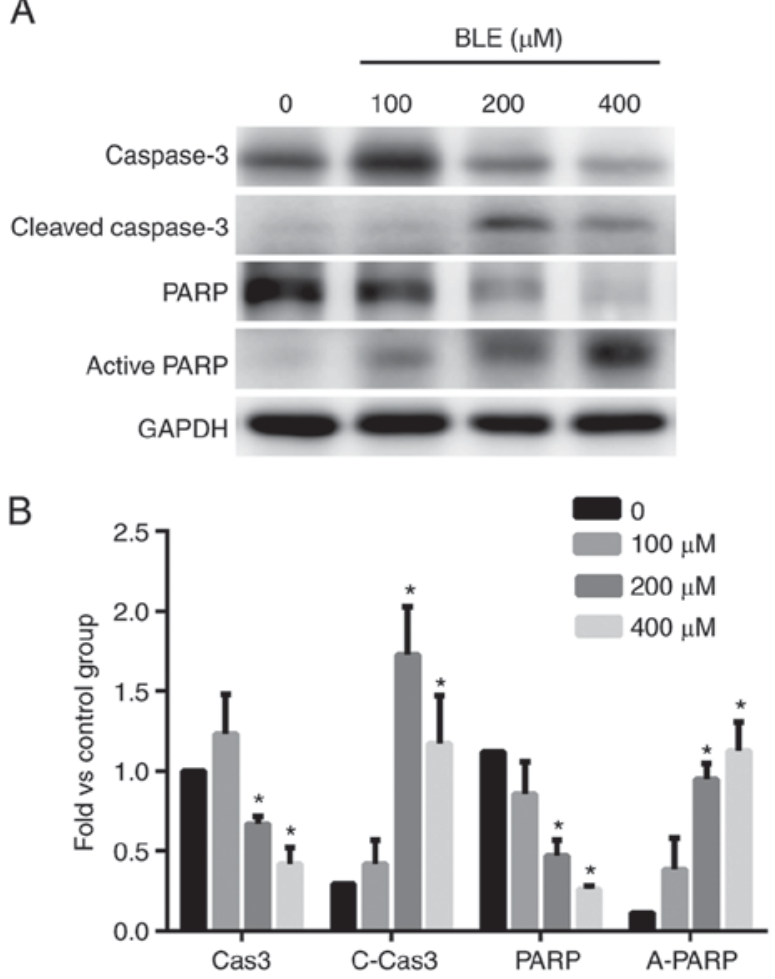

Figure 5. (A) Western blot analysis of downstream cell apoptotic signaling pathways proteins in NPDFs at $0,100,200$, or $400 \mu \mathrm{M}$ BLE for $48 \mathrm{~h}$. (B) Statistical analysis of A. ${ }^{*} \mathrm{P}<0.05$. NPDFs, nasal polyp-derived fibroblasts.

exhibited increased cell apoptosis in concentration-dependent manner. These results further confirmed that bleomycin A5 can induce NPDF apoptosis in a dose-dependent manner.

Caspases, PARP, and Bcl-2 family participate in bleomycin A5-induced apoptosis in NPDFs. We next focused on identifying the pathways that lead to NPDF apoptosis using bleomycin A5. To identify the genes involved in apoptosis, we first conducted a PCR array using NPDFs treated with bleomycin A5 for $48 \mathrm{~h}$ at different concentrations. Using RT-qPCR, the mRNA levels of Bcl-2, Bax, and caspase-3 increased, and the difference was statistically significant $(\mathrm{P}<0.05)$ after bleomycin A5 treatment for $48 \mathrm{~h}$ (Fig. 4).

Next, we examined the changes in the protein levels of these genes using western blot analysis. Even though the
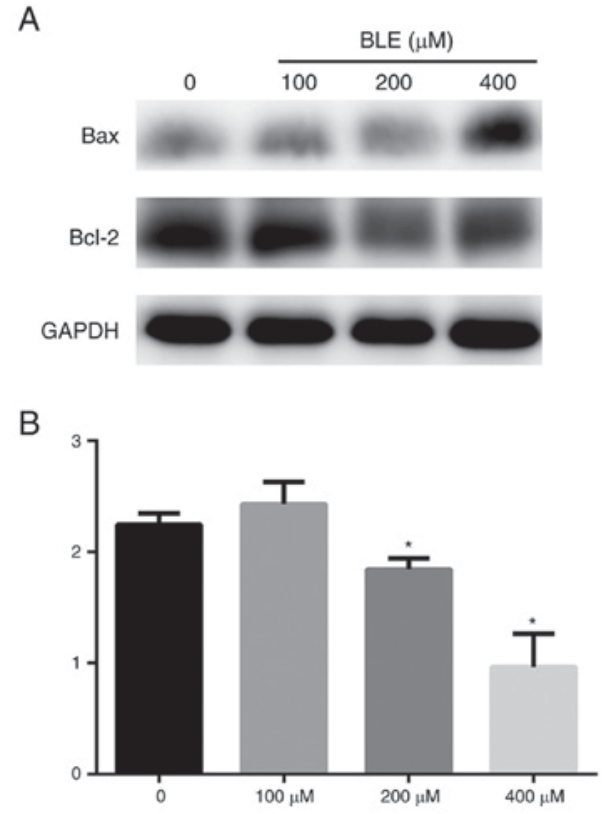

Figure 6. (A) Western blot analysis of mitochondrial apoptosis-related proteins in NPDFs at $0,100,200$, or $400 \mu \mathrm{M}$ BLE for $48 \mathrm{~h}$. (B) Statistical analysis of ratio of $\mathrm{Bcl}-2 / \mathrm{Bax}$ of $\mathrm{A} .{ }^{*} \mathrm{P}<0.05$. NPDFs, nasal polyp-derived fibroblasts.

$100 \mu \mathrm{M}$ group seemed to show more caspase- 3 expression than the control group, the difference was not significant. However, the levels of cleaved caspase- 3 increased in a dose-dependent manner (Fig. 5). The levels of PARP, a zymolyte of cleaved caspase-3, also declined as the concentration of bleomycin A5 increased, and the level of active PARP increased in a dose-dependent manner. These results indicated that the concentrations of the apoptotic downstream effective proteins were significantly higher in both the 200 and $400 \mu \mathrm{M}$ groups than in the control group (Fig. 5).

Bcl-2 and Bax are 2 important mitochondria-mediated caspase-dependent apoptotic proteins. It has been proven that Bax forms a complex with the anti-apoptotic protein $\mathrm{Bcl}-2$ to balance its apoptotic effects (15). The ratio of $\mathrm{Bcl}-2 / \mathrm{Bax}$ was used to determine whether cells undergo programmed cell death. The Bcl-2/Bax ratio was determined through densitometry and normalized to the GAPDH levels of each group. The results indicated that stimulation of NPDFs with bleomycin A5 in vitro resulted in a dose-dependent decrease in the Bcl-2/Bax ratio (Fig. 6). To further confirm this conclusion, pro-apoptotic protein Bax was knockdown by siRNA transfection. The Annexin V/PI staining shows that such knockdown could significantly attenuate apoptosis in BLE-treated NPDFs (Fig. 7).

\section{Discussion}

Recent studies have shown that cultures of human dermal fibroblasts undergo reductions in lysyl oxidase concentration as a result of the action of bleomycin. In addition, fibroblast apoptosis and proliferation levels are both higher during bleomycin-induced lung fribrosis (16). Results have demonstrated that bleomycin, which mainly targets the fibroblasts in scar tissue, can induce keloid fibroblast death 
A

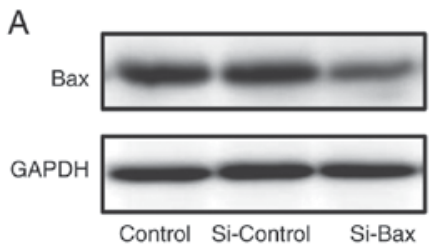

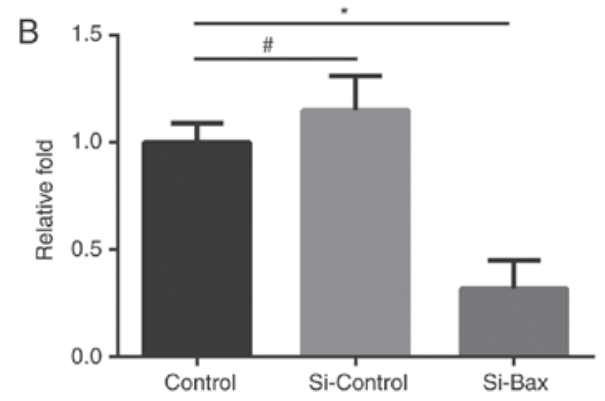
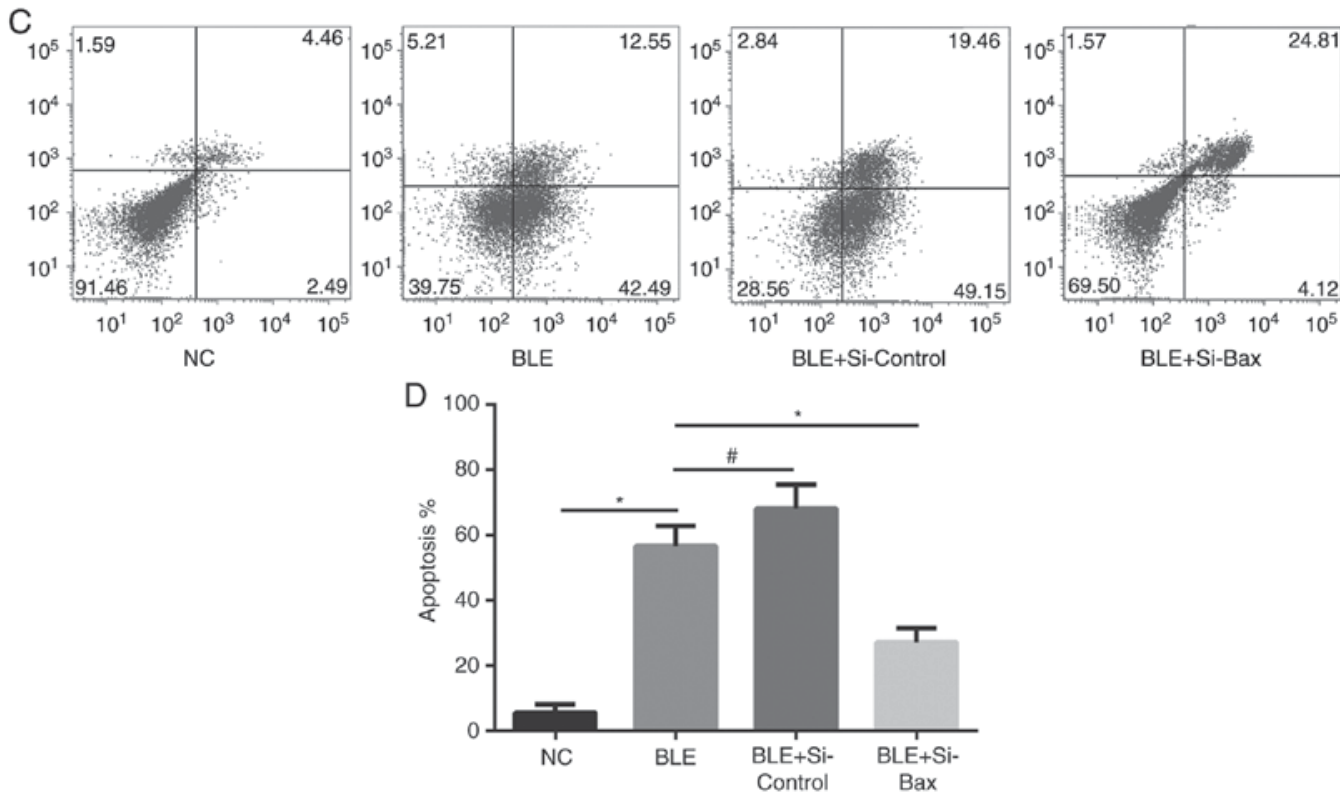

Figure 7. (A) Western blot analysis of Bax expression in NPDFs transfected with siRNA or si-Control. (B) Statistical analysis of A. (C) Apoptotic analysis by Annexin V/PI staining of the transfected NPDFs. (D) Statistical analysis of C. The results are here expressed as the mean \pm standard deviation ( $\mathrm{n}=3$ ), ${ }^{*} \mathrm{P}<0.05$. NPDFs, nasal polyp-derived fibroblasts.

and lead to a reduction in collagen synthesis. For this reason, the intralesional injection of bleomycin A5 into keloids and hypertrophic scars has been proposed as a modality for scar treatment and scar prevention after surgery (17). Because of the effects of bleomycin on fibroblasts and its roles in the production of cytokines and collagen in stromal fibroblasts in nasal polyp tissue, we here propose that bleomycin A5 may have an inhibitory effect on NPDFs. In fact, when we performed this study, we also collected clinical data regarding the use of intralesional bleomycin A5 in nasal polyps, and results showed that this off-label use can cure nasal polyps and improve the nasal ventilation function of patients suffering from nasal polyps effectively (data not shown). In this study, we further isolated NPDFs from surgical specimens of nasal polyp tissue and then used a primary culture to investigate bleomycin A5-induced apoptosis in NPDFs. Results showed that bleomycin A5 induced apoptosis in NPDFs in a time- and concentration-dependent manner through activation of bcl-2 family and caspase cascades.

Previous studies have shown bleomycin A5-induced apoptosis in primary cultures of rat type II alveolar epithelial cells and in human A549 cells to be concentration- and time-dependent (18). To determine whether bleomycin-A5 induced NPDF apoptosis is time- or concentration-dependent in NPDFs, we choose different concentrations of bleomycin A5 and durations of stimulus. We found that at higher concentrations of bleomycin A5 and over longer periods of exposure, the number of NPDFs with condensed cytoplasm and nuclei increased and total number of cells decreased. Notably, at $48 \mathrm{~h}$, the number of cells undergoing apoptosis became stable regardless of concentration. For this reason, we choose $48 \mathrm{~h}$ for the rest of our experiments.

PARP is a DNA repair enzyme that is activated by various environmental stimuli, including ROS, free radicals, and peroxynitrite. During almost all forms of apoptosis, PARP can be processed in vivo into its apoptotic 24 and $89 \mathrm{kDa}$ fragments by caspase-3 or -7 (19). In this study, we found that bleomycin A5-treated NPDFs contained significantly greater amounts of the active forms of caspase-3 and underwent more cleavage of PARP at the protein levels. These results indicated that increased activation of caspase cascades mediated bleomycin A5 induced NPDF apoptosis.

Previous studies indicate that activation of the pro-apoptotic Bcl-2 family is required for the development of pulmonary fibrosis after the intratracheal instillation of bleomycin in mice. Mice lacking Bid exhibited significantly less pulmonary fibrosis in response to bleomycin than WT mice (20). It is here suggested that apoptosis mediated by members of the Bcl-2 family may play an essential role in apoptosis of lung cells (21). The bcl-2 family is divided into 2 groups: Pro-apoptotic and anti-apoptotic 
molecules. Pro-apoptotic proteins can induce apoptosis through breakage of the mitochondrial membrane potential (22). To the contrary, by binding to specific site of Bax, the anti-apoptotic proteins retained normal permeability and prevented the release of mitochondrial pro-apoptotic factors into the cytoplasm (23). Thus, the balance of pro-apoptotic and anti-apoptotic proteins is often used to regulate cell apoptosis (17). In this study, we analyzed the pro-apoptotic molecules Bax and the anti-apoptotic molecule Bcl-2 using RT-PCR and Western blotting. These results indicated that there was significantly more mRNA and protein expression of the pro-apoptotic Bax molecules in bleomycin A5-treated NPDFs than in untreated NPDFs. In contrast, expression of Bcl-2 mRNA and protein was significantly lower in bleomycin A5-treated NPDFs than in untreated NPDFs. And knockdown of Bax result in decrease of apoptosis in BLE-treated NPDFs. This pro-apoptotic change in the expression levels of members of the Bcl-2 family, which are major regulators of apoptosis via the intrinsic pathway, suggests that the induction of apoptosis in NPDFs by bleomycin A5 is mediated by an intrinsic mitochondria-mediated pathway.

In conclusion, this study demonstrated that bleomycin A5 induces apoptosis of primary NPDFs in association with increased expression of pro-apoptotic proteins, decreased expression of genes in the anti-apoptotic Bcl-2 family and increased expression of caspase cascades (caspase- 3 and PARP) in a time-, concentration-, and caspase-dependent manner. Intralesional injection has already been used in this way in clinical settings for the treatment of infantile hemangiomas, keloids, and hypertrophic scar, and this approach may be a suitable alterative therapeutic option for patients with nasal polyps, especially recurrent, difficult-to-treat, and glucocorticoid insensitive cases.

\section{Acknowledgements}

This study was supported by grants from National Natural Science Foundation of China (no. 81500773), the Natural Science Foundation of Guangdong Province of China (no. 2015A030310125), and the Natural Science Foundation of Guangdong Province of China (no. 2014A030313158); Grant [2013]163 from Key Laboratory of Malignant Tumor Molecular Mechanism and Translational Medicine of Guangzhou Bureau of Science and Information Technology; Grant KLB09001 from the Key Laboratory of Malignant Tumor Gene Regulation and Target Therapy of Guangdong Higher Education Institutes.

\section{References}

1. Thomas M, Yawn BP, Price D, Lund V, Mullol J and Fokkens W; European Position Paper on Rhinosinusitis and Nasal Polyps Group: EPOS primary care guidelines: European position paper on the primary care diagnosis and management of rhinosinusitis and nasal polyps 2007-a summary. Prim Care Respir J 17: 79-89, 2008.

2. Hedman J, Kaprio J, Poussa T and Nieminen MM: Prevalence of asthma, aspirin intolerance, nasal polyposis and chronic obstructive pulmonary disease in a population-based study. Int J Epidemiol 28: 717-722, 1999.

3. Wang QP, Escudier E, Roudot-Thoraval F, Abd-Al Samad I, Peynegre R and Coste A: Myofibroblast accumulation induced by transforming growth factor-beta is involved in the pathogenesis of nasal polyps. Laryngoscope 107: 926-931, 1997.
4. Park IH, Park SJ, Cho JS, Moon YM, Kim TH, Lee SH and Lee HM: Role of reactive oxygen species in transforming growth factor beta1-induced alpha smooth-muscle actin and collagen production in nasal polyp-derived fibroblasts. Int Arch Allergy Immunol 159: 278-286, 2012.

5. Park HH, Park IH, Cho JS, Lee YM and Lee HM: The effect of macrolides on myofibroblast differentiation and collagen production in nasal polyp-derived fibroblasts. Am J Rhinol Allergy 24: 348-353, 2010.

6. Shin JM, Park JH, Park IH and Lee HM: Doxycycline inhibits TGF- $\beta 1$-induced extracellular matrix production in nasal polyp-derived fibroblasts. Int Forum Allergy Rhinol 6: 256-263, 2016.

7. Ledon JA, Savas J, Franca K, Chacon A and Nouri K: Intralesional treatment for keloids and hypertrophic scars: A review. Dermatol Surg 39: 1745-1757, 2013.

8. Jones CD, Guiot L, Samy M, Gorman M and Tehrani H: The use of chemotherapeutics for the treatment of keloid scars. Dermatol Reports 7: 5880, 2015.

9. Hendricks T, Martens MF, Huyben CM and Wobbes T: Inhibition of basal and TGF beta-induced fibroblast collagen synthesis by antineoplastic agents. Implications for wound healing. Br J Cancer 67: 545-550, 1993.

10. Yeowell HN, Marshall MK, Walker LC, Ha V and Pinnell SR: Regulation of lysyl oxidase mRNA in dermal fibroblasts from normal donors and patients with inherited connective tissue disorders. Arch Biochem Biophys 308: 299-305, 1994.

11. Wallach-Dayan SB, Izbicki G, Cohen PY, Gerstl-Golan R, Fine A and Breuer R: Bleomycin initiates apoptosis of lung epithelial cells by ROS but not by Fas/FasL pathway. Am J Physiol Lung Cell Mol Physiol 290: L790-L796, 2006.

12. Wang R, Ibarra-Sunga O, Verlinski L, Pick R and Uhal BD: Abrogation of bleomycin-induced epithelial apoptosis and lung fibrosis by captopril or by a caspase inhibitor. Am J Physiol Lung Cell Mol Physiol 279: L143-L151, 2000.

13. Zhang X, Zou J, Li B, Ren X and Shi J: Eosinophil apoptosis in nasal polyposis tissue after bleomycin A5 local injection. Lin Chuang Er Bi Yan Hou Ke Za Zhi 18: 279-281, 2004 (In Chinese).

14. Park IH, Um JY, Hong SM, Cho JS, Lee SH, Lee SH and Lee HM: Metformin reduces TGF- $\beta 1$-induced extracellular matrix production in nasal polyp-derived fibroblasts. Otolaryngol Head Neck Surg 150: 148-153, 2014.

15. Sharifi S, Barar J, Hejazi MS and Samadi N: Roles of the $\mathrm{Bcl}-2 / \mathrm{Bax}$ ratio, caspase- 8 and 9 in resistance of breast cancer cells to paclitaxel. Asian Pac J Cancer Prev 15: 8617-8622, 2014.

16. Tsukui T, Ueha S, Abe J, Hashimoto S, Shichino S, Shimaoka T, Shand FH, Arakawa Y, Oshima K, Hattori M, et al: Qualitative rather than quantitative changes are hallmarks of fibroblasts in bleomycin-induced pulmonary fibrosis. Am J Pathol 183: 758-773, 2013.

17. Wang XQ, Liu YK, Qing C and Lu SL: A review of the effectiveness of antimitotic drug injections for hypertrophic scars and keloids. Ann Plast Surg 63: 688-692, 2009.

18. Li X, Zhang H, Soledad-Conrad V, Zhuang J and Uhal BD: Bleomycin-induced apoptosis of alveolar epithelial cells requires angiotensin synthesis de novo. Am J Physiol Lung Cell Mol Physiol 284: L501-L507, 2003.

19. Bleackley RC and Heibein JA: Enzymatic control of apoptosis. Nat Prod Rep 18: 431-440, 2001.

20. Budinger GR, Mutlu GM, Eisenbart J, Fuller AC, Bellmeyer AA, Baker CM, Wilson M, Ridge K, Barrett TA, Lee VY and Chandel NS: Proapoptotic Bid is required for pulmonary fibrosis. Proc Natl Acad Sci USA 103: 4604-4609, 2006.

21. Kasper M and Barth $\mathrm{K}$ : Bleomycin and its role in inducing apoptosis and senescence in lung cells-modulating effects of caveolin-1. Curr Cancer Drug Targets 9: 341-353, 2009.

22. Chen YB, Aon MA, Hsu YT, Soane L, Teng X, McCaffery JM, Cheng WC, Qi B, Li H, Alavian KN, et al: Bcl-xL regulates mitochondrial energetics by stabilizing the inner membrane potential. J Cell Biol 195: 263-276, 2011.

23. Prenek L, Boldizsár F, Kugyelka R, Ugor E, Berta G, Németh P and Berki T: The regulation of the mitochondrial apoptotic pathway by glucocorticoid receptor in collaboration with Bcl-2 family proteins in developing $\mathrm{T}$ cells. Apoptosis 22: 239-253, 2017. 